\title{
Electrothermally driven high-frequency piezoresistive SiC cantilevers for dynamic atomic force microscopy
}

R. Boubekri, E. Cambril, L. Couraud, L. Bernardi, A. Madouri, M. Portail, T. Chassagne, C. Moisson, M. Zielinski, S. Jiao, J.-F. Michaud, D. Alquier, J. Bouloc, L. Nony, F. Bocquet, C. Loppacher, D. Martrou, and S. Gauthier

Citation: Journal of Applied Physics 116, 054304 (2014); doi: 10.1063/1.4891833

View online: https://doi.org/10.1063/1.4891833

View Table of Contents: http://aip.scitation.org/toc/jap/116/5

Published by the American Institute of Physics

\section{Articles you may be interested in}

Fundamental piezoresistive coefficients of p-type single crystalline 3C-SiC

Applied Physics Letters 104, 111905 (2014); 10.1063/1.4869151

Analysis of piezoresistance in $\mathrm{n}$-type $\beta$-SiC for high-temperature mechanical sensors

Applied Physics Letters 81, 2797 (2002); 10.1063/1.1513652

Atomic resolution with an atomic force microscope using piezoresistive detection

Applied Physics Letters 62, 834 (1993); 10.1063/1.108593

Micromachined atomic force microscopy sensor with integrated piezoresistive sensor and thermal bimorph actuator for high-speed tapping-mode atomic force microscopy phase-imaging in higher eigenmodes Journal of Vacuum Science \& Technology B: Microelectronics and Nanometer Structures Processing, Measurement, and Phenomena 21, 3102 (2003); 10.1116/1.1614252

Electrothermal actuation of silicon carbide ring resonators Journal of Vacuum Science \& Technology B: Microelectronics and Nanometer Structures Processing, Measurement, and Phenomena 27, 3109 (2009); 10.1116/1.3244622

Factors affecting the $\mathrm{f} \times \mathrm{Q}$ product of $3 \mathrm{C}$-SiC microstrings: What is the upper limit for sensitivity? Journal of Applied Physics 119, 055304 (2016); 10.1063/1.4941274

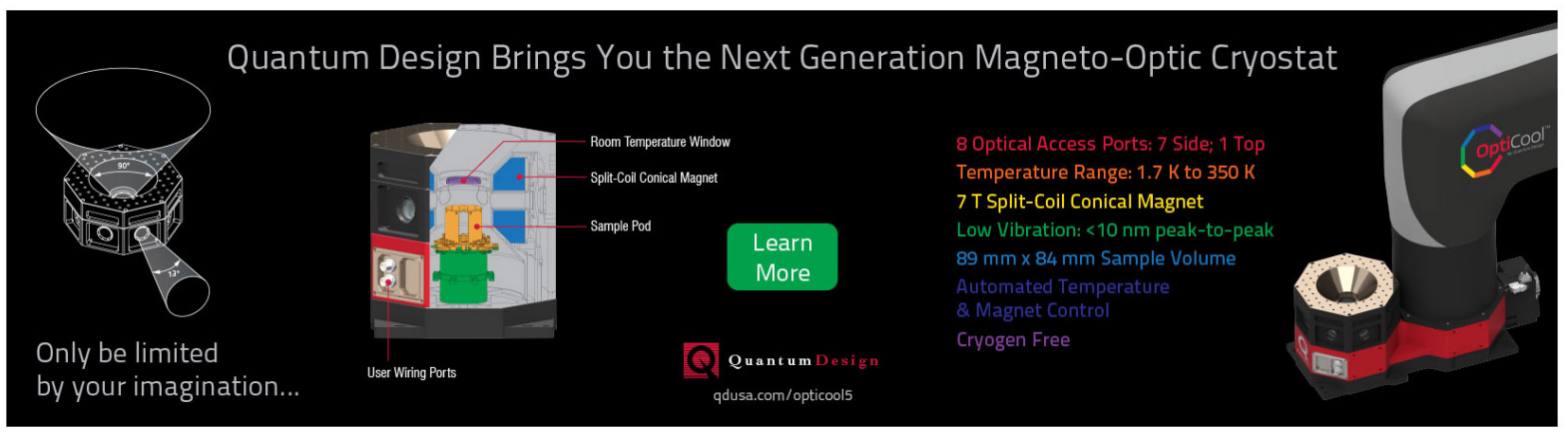




\title{
Electrothermally driven high-frequency piezoresistive SiC cantilevers for dynamic atomic force microscopy
}

\author{
R. Boubekri, ${ }^{1}$ E. Cambril, ${ }^{1}$ L. Couraud,${ }^{1}$ L. Bernardi, ${ }^{1}$ A. Madouri, ${ }^{1}$ M. Portail, ${ }^{2}$ \\ T. Chassagne,${ }^{3}$ C. Moisson, ${ }^{3}$ M. Zielinski, ${ }^{3}$ S. Jiao, ${ }^{4}$ J.-F. Michaud, ${ }^{4}$ D. Alquier, ${ }^{4}$ J. Bouloc, ${ }^{5}$ \\ L. Nony, ${ }^{5}$ F. Bocquet ${ }^{5}$ C. Loppacher, ${ }^{5}$ D. Martrou, ${ }^{6}$ and S. Gauthier ${ }^{6, a)}$ \\ ${ }^{1} L P N$, CNRS-UPR20, route de Nozay, 91460 Marcoussis, France \\ ${ }^{2}$ Centre de Recherche sur l'Hetero-Epitaxie et ses Applications CNRS, Rue Bernard Gregory, \\ 06560 Valhonne, France \\ ${ }^{3}$ NOVASiC, Savoie Technolac, Arche bât 4, BP 267, 73375 Le Bourget du Lac Cedex, France \\ ${ }^{4}$ Université François Rabelais, Tours, GREMAN, CNRS-UMR7347, 16 rue Pierre et Marie Curie, BP 7155, \\ 37071 Tours Cedex 2, France \\ ${ }_{5}^{5}$ IM2NP-CNRS/Aix-Marseille University, 38 rue Frédéric Joliot-Curie, 13451 Marseille, France \\ ${ }^{6}$ CEMES, CNRS UPR 8011 et Université de Toulouse, 29 rue Jeanne Marvig, 31055 Toulouse, France
}

(Received 15 July 2014; accepted 21 July 2014; published online 5 August 2014)

\begin{abstract}
Cantilevers with resonance frequency ranging from $1 \mathrm{MHz}$ to $100 \mathrm{MHz}$ have been developed for dynamic atomic force microscopy. These sensors are fabricated from 3C-SiC epilayers grown on $\mathrm{Si}(100)$ substrates by low pressure chemical vapor deposition. They use an on-chip method both for driving and sensing the displacement of the cantilever. A first gold metallic loop deposited on top of the cantilever is used to drive its oscillation by electrothermal actuation. The sensing of this oscillation is performed by monitoring the resistance of a second Au loop. This metallic piezoresistive detection method has distinct advantages relative to more common semiconductor-based schemes. The optimization, design, fabrication, and characteristics of these cantilevers are discussed. (C) 2014 AIP Publishing LLC. [http://dx.doi.org/10.1063/1.4891833]
\end{abstract}

\section{INTRODUCTION}

Except at its very beginning, Atomic Force Microscopy (AFM) has been dependent on micro-fabricated force sensors. Nowadays, most AFM work with Si nitride or Si cantilevers and use optical detection techniques (beam deflection in most cases ${ }^{1,2}$ but also interferometry ${ }^{3}$ ). Electrical detection techniques based on piezoresistive $e^{4-7}$ or piezoelectric ${ }^{8,9}$ cantilevers have been also developed and employed, but to a lesser extent. Piezoelectric quartz-based sensors are becoming popular, especially for low temperature dynamic AFM studies. ${ }^{10-12}$ But despite all these developments, dynamic AFM is still confined to relatively low frequency ( $\leqslant 1 \mathrm{MHz}$ ), while MEMS and Nano-Electro-Mechanical Systems (NEMS) working at frequencies as high as $1 \mathrm{GHz}$ have been already demonstrated. ${ }^{13}$

There is much to gain in increasing the frequency of AFM force sensors. An increase in the resonant frequency should lead to a better signal-to-noise ratio and a larger acquisition bandwidth. Indeed, AFM heads dedicated to fast imaging have been developed with great success, ${ }^{14}$ opening the possibility to fully exploit the benefits expected from high frequency cantilevers.

Increasing the frequency means reducing the size of the device, which in turn imposes to develop new actuation and detection schemes. It was recently proposed ${ }^{15}$ to use a metallic piezoresistance to follow the oscillation of a cantilever. Despite the fact that the piezoresistive gauge factor of metals is several orders of magnitude lower than the gauge factor of

${ }^{\text {a)} E l e c t r o n i c ~ m a i l: ~ g a u t h i e r @ c e m e s . f r ~}$ doped semiconductors, which have been generally used in piezoresistive motion detection, ${ }^{4-7}$ this new approach could be advantageous. The signal-to-noise ratio is not degraded as much as the ratio of the gauge factors would imply because doped semiconductors exhibit much larger $1 / \mathrm{f}$ noise than metals. In addition, due to the lower resistivity of metals, it is easier to adjust the value of the piezoresistance to $50 \Omega$, avoiding the necessity of impedance matching with the readout device. Finally, using a metal greatly simplifies the fabrication process.

The flexural resonance frequency of a "diving board" cantilever is proportional to the velocity of sound in the material $V=\left(\frac{E}{\rho}\right)^{\frac{1}{2}}$, where $E$ is the Young's modulus and $\rho$ is the density of the material. This makes $\mathrm{SiC}$ a good material to increase the resonance frequency with its larger $V$ when compared to Si (see Table I). The cantilevers developed in this work were made by surface micromachining $3 \mathrm{C}-\mathrm{SiC}$ films grown on $\mathrm{Si}$ wafers according to established techniques. ${ }^{16,17}$

Each AFM mode has its own cantilever specifications. The target here is to optimize cantilevers with resonant frequencies as high as $100 \mathrm{MHz}$ for the most recent of these modes: non-contact AFM (nc-AFM) or frequency modulation AFM (FM-AFM). ${ }^{18}$

The paper is organized as follows: An expression of the measurement noise of piezoresistive cantilevers is derived in Sec. II. This expression is used in Sec. III to optimize the design of the cantilevers. The fabrication of the cantilevers is detailed in Sec. IV. The measurement of the resonance properties of the electrothermally driven cantilevers is presented in Sec. V. This part is followed by a conclusion (Sec. VI). 
TABLE I. Material properties used to calculate the values given in Figure 3. The value of the shear modulus of $\mathrm{SiC}$ is approximated by $G=\frac{E}{2(1+\nu)}$ which is the expression for an isotropic medium, $\nu$ is the Poisson's ratio.

\begin{tabular}{lccccc}
\hline \hline Material & $\mathrm{E}(\mathrm{GPa})$ & $\nu$ & $\mathrm{G}(\mathrm{GPa})$ & $\rho\left(\mathrm{g} \cdot \mathrm{cm}^{-3}\right)$ & $V=\left(\frac{E}{\rho}\right)^{\frac{1}{2}}\left(\mathrm{~m} \cdot \mathrm{s}^{-1}\right)$ \\
\hline $\mathrm{Si}$ & $169^{26}$ & 0.42 & $50^{26}$ & $2.33^{26}$ & 8517 \\
$\mathrm{Au}$ & $78^{15}$ & 0.42 & & & \\
3C-SiC & $440^{15}$ & $0.22^{27}$ & $\sim 180$ & $3.166^{15}$ & 11789 \\
\hline \hline
\end{tabular}

\section{MEASUREMENT NOISE OF PIEZORESISTIVE CANTILEVERS}

As for any scientific instrument, the precision of ncAFM measurements is limited by noise. This noise can be traced back to several noise sources. The most fundamental of these sources is the thermomechanical noise of the cantilever, which has been considered in numerous works. ${ }^{19-21}$ Another important source is the measurement noise, which is specific to the method chosen to measure the displacement of the cantilever. ${ }^{18,20}$ There are also external noise sources such as building vibrations or temperature fluctuations. ${ }^{22}$

It is beyond the scope of the present work to discuss in detail the impact of these noise sources on AFM data. Here, we take for granted the main conclusions of previous works ${ }^{18,23}$ that led to the conclusion that the measurement noise is minimized when the oscillation amplitude is of the order of magnitude of the decay length of the tip-surface interaction, meaning $\mathrm{A} \simeq 100 \mathrm{pm}$ for a typical short-range interaction potential, and the cantilever stiffness $k$ is of the order of $1000 \mathrm{~N} \cdot \mathrm{m}^{-1}$, mainly to prevent jump to contact instabilities. We then use the minimization of the measurement noise associated with the piezoresistive method as an optimization criterion for our cantilevers.

The principle of a piezoresistive cantilever is to use the variations $\Delta R$ of the value of the piezoresistance $R$ to monitor the displacement of the cantilever extremity $\Delta z$ according to $\Delta R / R=\eta \Delta z$, where $\eta$ is the sensitivity of the cantilever. An upper bound for $\eta$ can be derived easily. In a piezoresistance, $\Delta R$ is related to the strain $\varepsilon$ by the gauge factor $\gamma$

$$
\gamma=\frac{\Delta R}{\epsilon R} .
$$

The strain in a cantilever whose extremity is displaced by $\Delta z$ is given by ${ }^{24}$

$$
\epsilon(y, c)=-\frac{3(y-L) c}{L^{3}} \Delta z
$$

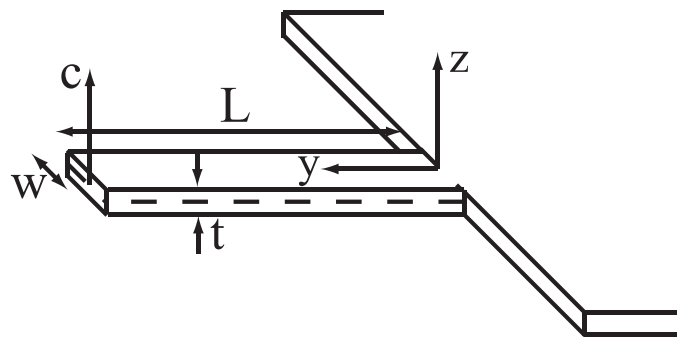

FIG. 1. Geometry of the cantilevers. where $\mathrm{c}$ is the distance to the neutral plane, in the middle of the cantilever (Figure 1). The strain is maximal at the base $(y=0)$ and at the surface of the cantilever $(c=t / 2)$

$$
\varepsilon_{\max }=\frac{3 t}{2 L^{2}} \Delta z
$$

hence

$$
\left(\frac{\Delta R}{R}\right)_{\max }=\gamma \epsilon_{\max }=\frac{3 \gamma t}{2 L^{2}} \Delta z
$$

and

$$
\eta_{\max }=\left(\frac{\Delta R}{R}\right)_{\max } / \Delta z=\frac{3 \gamma t}{2 L^{2}} .
$$

To maximize $\eta$, it is necessary to concentrate the piezoresistance near the base of the cantilever. Note that the resonance frequency of the first flexural mode is given by

$$
f_{0} \approx \frac{1}{2 \pi}\left(\frac{E}{\rho}\right)^{\frac{1}{2}} \frac{t}{L^{2}} .
$$

It depends on the cantilever dimension in the same way as $\eta_{\max }$ and one can write

$$
\eta_{\max } \approx 3 \pi \gamma\left(\frac{\rho}{E}\right)^{1 / 2} f_{0}
$$

meaning that the sensitivity of the piezoresistive cantilever increases linearly with its resonance frequency. This dependence makes this detection technique particularly efficient at high frequency.

If the value of the cantilever piezoresistance is adjusted to $50 \Omega$, a direct (i.e., without impedance matching) measurement method is possible, as illustrated in Figure 2. The piezoresistance $R$ is polarized by $\mathrm{V}_{\mathrm{DC}}$. The RF variations of the piezoresistance $\Delta R / R$, induced by the cantilever oscillation, generate a RF voltage across $R$ that reads

$$
\Delta V_{R F}=I_{D C} \Delta R=V_{D C} \Delta R / R=V_{D C} \eta \Delta z .
$$

This voltage is applied to the input of the $R F$ voltage amplifier via the $\mathrm{dc} / R F$ bias tee to give the output signal

$$
\Delta V_{\text {out }}=G_{p} \Delta V_{R F},
$$

where $G_{p}$ is the amplifier voltage gain.

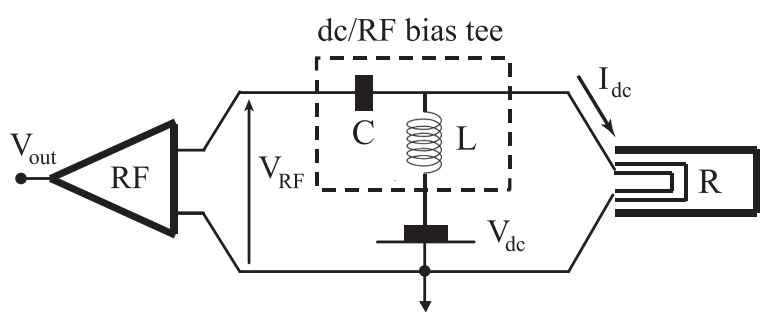

FIG. 2. Principle of the piezoresistance measurement method. 
This measurement method introduces two noise sources:

- The Nyquist-Johnson noise of the piezoresistance characterized by its power spectral density (PSD): $S_{V}(\omega)$ $=4 k_{B} T R\left(\right.$ in $\left.\mathrm{V}^{2} \mathrm{~Hz}^{-1}\right)$.

- The amplifier noise, with the PSD: $S_{V i n}\left(\right.$ in $\left.\mathrm{V}^{2} \mathrm{~Hz}^{-1}\right)$.

It is customary to refer the noise to the input of the device under consideration, in our case the deflection sensor. One then gets the PSD of the cantilever deflection due to the measurement noise

$$
S_{z}(\omega)=\frac{1}{\left(\eta V_{D C}\right)^{2}}\left(4 k_{B} T R+S_{V i n}\right)\left(i n \mathrm{~m}^{2} \mathrm{~Hz}^{-1}\right)
$$

or if $\eta \approx \eta_{\max }$

$$
S_{z}(\omega) \approx \frac{E}{\rho} \frac{1}{\left(3 \pi \gamma f_{0} V_{D C}\right)^{2}}\left(4 k_{B} T R+S_{V i n}\right) .
$$

To minimize this contribution, it is necessary to

- Maximize $\mathrm{V}_{\mathrm{DC}}$, with a limit given by the temperature rise of the cantilever induced by the power dissipated by Joule effect in the piezoresistor. In the following, we use $V_{D C}=0.1 \mathrm{~V}$, corresponding to $P=V_{D C}^{2} / R=0.2 \mathrm{~mW}$.

- Minimize $S_{V i n}$. For a good RF amplifier, ${ } S_{V i n}$ $\approx 1 \mathrm{nV} \cdot \mathrm{Hz}^{-1 / 2}$, which is of the same order of magnitude as $\sqrt{ }\left(4 k_{B} T R\right) \approx 0.9 \mathrm{nV} . \mathrm{Hz}^{-1 / 2}$ (at room temperature and for $R=50 \Omega$ ). Reducing the noise level of the amplifier beyond this value is not very useful at room temperature, but becomes crucial at low temperature.

- Maximize $\eta$ by positioning the piezoresistance in the region of maximal stress.

- Maximize the piezoelectric gauge factor $\gamma$.

The different optimization parameters at hand are listed in Table II. Note that the requirement of an as-large-as-possible quality factor $\mathrm{Q}$ stems from the minimization of the thermomechanical noise of the cantilever. ${ }^{19,20}$

\section{DESIGN OF THE CANTILEVERS}

Our goal was to fabricate a family of cantilevers, with resonance frequencies ranging from $1 \mathrm{MHz}$ to $100 \mathrm{MHz}$.

\section{A. Mechanical design}

It was chosen to fabricate all these cantilevers from $\mathrm{SiC}$ films of the same thickness to avoid the complication involved in preparing wafers with SiC films of different thicknesses. The flexural resonance frequencies of a "diving

TABLE II. Optimization parameters. $f_{0}$ : resonance frequency, $k$ : stiffness, $Q$ : quality factor, $\gamma$ : piezoresistive gauge factor, $\eta$ : sensitivity, $R$ : resistance of the piezoresistive circuit, $V_{\mathrm{DC}}$ : bias voltage applied to the piezoresistive

\begin{tabular}{|c|c|c|c|c|c|c|}
\hline$f_{0}(\mathrm{MHz})$ & $k(\mathrm{~N} / \mathrm{m})$ & $\mathrm{Q}, \gamma$ & $\eta$ & $\mathrm{R}(\Omega)$ & $V_{\mathrm{DC}}(V)$ & $S_{\text {Vin }}\left(\mathrm{nV} \cdot \mathrm{Hz}^{-1 / 2}\right)$ \\
\hline$\approx 100$ & $\sim 1000$ & $\begin{array}{c}\text { As large as } \\
\text { possible }\end{array}$ & $\lesssim 3 \gamma t / 2 L^{2}$ & $\approx 50$ & $\leq 0.1$ & $\approx 1$ \\
\hline
\end{tabular}
circuit, $S_{\mathrm{Vin}}$ : PSD of the amplifier noise. board" cantilever of length $L$, width $w$, and thickness $t$ are given by ${ }^{24}$

$$
f_{n}=\frac{\alpha_{n}}{2 \pi}\left(\frac{E}{\rho}\right)^{\frac{1}{2}} \frac{t}{L^{2}}, \quad \alpha_{1}=1.01, \quad \alpha_{2}=6.36, \quad \alpha_{3}=17.81,
$$

while the stiffness is given by ${ }^{24}$

$$
k=\frac{E w t^{3}}{4 L^{3}} .
$$

It is important to ensure that the first flexural resonance of the cantilever is well separated from its other modes, in order to avoid spurious couplings that could lead to an illdefined oscillation. Expression (6) shows that the diving board geometry naturally satisfies this requirement for the flexural modes. The first torsional resonance frequency is given by ${ }^{25}$ $f_{T}=\frac{1}{4 L}\left(\frac{G J}{\rho I_{p}}\right)^{\frac{1}{2}}$ with $J \approx \frac{1}{3} w t^{3}\left[1.0-0.63 \frac{t}{w}+0.052\left(\frac{t}{w}\right)^{5}\right]$ and $I_{p}=\frac{1}{12}\left(t w^{3}+w t^{3}\right)$ where $G$ is the shear modulus. The resonance frequency and the stiffness divided by the width $w$ are shown as a function of $L$ in Figure 3(a). The torsional resonance frequency times the length $L$ as a function of $w$ is displayed in Fig. 3(b). These graphs have been calculated with the values given in Table I.

A careful analysis of these data shows that the larger thickness $t=500 \mathrm{~nm}$ is the best choice. In particular, it is clearly seen that it is not possible to reach high frequencies (100 MHz) while keeping the cantilever dimensions large enough to accommodate the piezoresistive circuit with smaller thickness $t$.

The calculated characteristics of cantilevers designed with $t=500 \mathrm{~nm}$ are displayed in Table III. Note that these dimensions are markedly different from the dimensions of the cantilevers fabricated in Ref. 15, especially concerning the thickness, which is here 5 times larger $(500 \mathrm{~nm}$ instead of $100 \mathrm{~nm}$ ). The reason is that the cantilevers of Ref. 15 were designed for mass sensing, where the stiffness should be kept as small as possible. As discussed in Sec. II, the constraints on the stiffness in nc-AFM applications are not the same and they lead to much higher values that authorize this increase of the thickness.

The stiffness for the two highest frequency cantilevers is in the range that was chosen in Sec. II. For all the cantilevers, the first torsional resonance frequency is at least three times higher than the first flexural resonance frequency, ensuring an efficient decoupling between these modes.

\section{B. Piezoresistance design}

The piezoelectric strain gauge factor is the sum of two contributions: $\gamma=\frac{\Delta R}{\epsilon R}=(1+2 \nu)+\frac{1}{\rho} \frac{\partial \rho}{\partial \epsilon}$. The first, purely geometric term, amounts to 1.84 for Au. The second, physical term, involves several mechanisms whose contribution depends on bulk properties but also on the microstructure of the piezoresistive film. It is largely dominant for doped semiconductors, but much smaller for metals. It is not possible to predict its precise value since it depends on the growth conditions of the metallic film. Nevertheless, measurements ${ }^{28}$ 


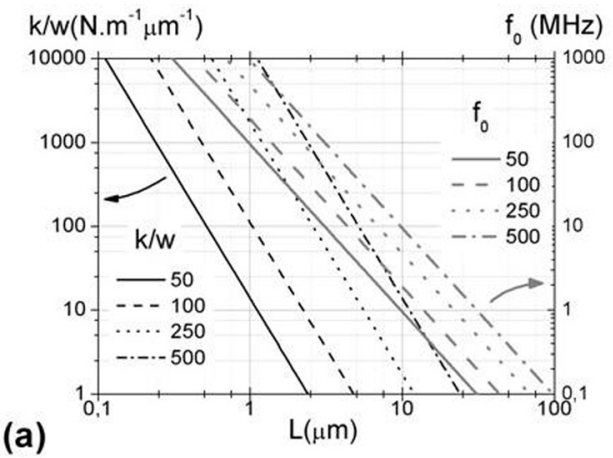

(b)

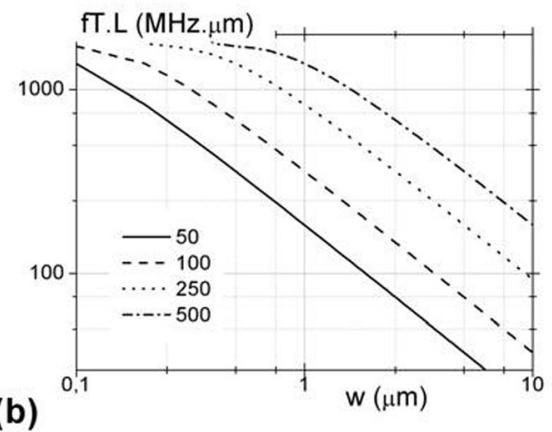

FIG. 3. (a) Resonance frequency (grey lines) and stiffness (black lines) divided by the width $w$ as a function of $L$ for different thicknesses t. (b) Torsional resonance frequency times the length $L$ as a function of w for different thicknesses $t$. suggest that it is of the same order of magnitude as the first term, giving $\gamma \sim 3$.

As mentioned in Ref. 15, using metallic piezoresistivity allows to design piezoresistive films with a resistance $R$ adjusted to $50 \Omega$, the standard input impedance of most high frequency readout devices, even for the smallest cantilevers considered in this work. $R$ depends not only on the geometry of the metallic path but also on the resistivity of the metal. It is well known that the resistivity of a metal increases relative to its bulk value for thin films, but its precise value will depend here again on the growth conditions of the metallic film.

On the basis of the measurements performed in Ref. 28, a thickness of $30 \mathrm{~nm}$ and a conductivity $\sigma=\sigma_{A u} / 3$ $\left(\sigma_{A u}=4.5 \times 10^{7} \mathrm{~S} \cdot \mathrm{m}^{-1}\right)$ were chosen for the design of the piezoresistive circuit. As explained in Sec. IV, the thickness of the film will be calibrated during the fabrication process to reach this value of $50 \Omega$. The circuit was positioned at the base of the cantilever, in the region where the strain is maximal, to enhance the sensitivity of the piezoelectric detection.

\section{Excitation method}

Many methods have been devised for exciting the cantilever vibration in dynamic AFM. The choice depends mainly on the environment and the characteristics of the cantilevers. The most common actuation method for dynamic AFM uses an external piezoelectric ceramics to drive the cantilever. It is simple to implement and give satisfactory results for low frequency and in-vacuum or in-air applications. At high frequency, it is preferable to use methods where the driving device is implemented on the cantilever to avoid spurious resonances due to the cantilever holder, which can introduce artefacts as demonstrated for instance in Ref. 30. Quartz sensors such as tuning forks ${ }^{11}$ or needle sensors ${ }^{10}$ can use the

TABLE III. Characteristics of SiC cantilevers designed with $t=500 \mathrm{~nm}$ and experimentally determined values of the resonance frequency $f_{\exp }$ and quality factor $Q$ for a family of cantilevers, $w, L$ : cantilever width and length, $f_{1}$ : flexural resonance frequency, $k$ : stiffness, $f_{T}$ : torsional resonance frequency.

\begin{tabular}{lcccccc}
\hline \hline$w(\mu \mathrm{m})$ & $L(\mu \mathrm{m})$ & $f_{1}(\mathrm{MHz})$ & $k(\mathrm{~N} / \mathrm{m})$ & $f_{T}(\mathrm{MHz})$ & $f_{\text {exp }}(\mathrm{MHz})$ & $Q$ \\
\hline 1.2 & 2.5 & 152 & 1056 & 498 & 112 & 660 \\
1.2 & 4 & 59 & 258 & 311 & $30-35$ & 2900 \\
2 & 7 & 19.3 & 80 & 120 & 15 & 8600 \\
5 & 14 & 4.83 & 25 & 26 & $4-5.2$ & 14500 \\
6 & 23 & 1.79 & 6.78 & 13 & $1.1-1.3$ & 11000 \\
\hline \hline
\end{tabular}

piezoelectricity of the material. Piezoelectric thin films (e.g., $\mathrm{ZnO}^{9}$ or titanates ${ }^{8}$ ) fabricated on top of the cantilever are also commonly used. Photothermal excitation, ${ }^{29}$ where an intensity modulated laser heats the cantilever at the right frequency, gives very good results especially in liquid, where getting a clean excitation is particularly critical. ${ }^{30}$

In electrothermal excitation, ${ }^{31,32}$ an alternative current is injected in a resistive circuit implemented on the cantilever. The inhomogeneity of the heating and the difference between the thermal expansion coefficient of the materials constituting the resistive circuit and the cantilever induce a bending of the cantilever. This method is well suited to our cantilevers. It is simple to implement because the resistive loop can be made in the same fabrication step and with the same material as the piezoresistive circuit. It has also the advantage of introducing a frequency doubling between the driving voltage and the piezoresistive response, facilitating the extraction of the displacement signal. One of its drawbacks is that it induces a temperature elevation of the cantilever. This problem is related to the efficiency of the driving. In the simplest models, ${ }^{33,39}$ the cantilever is considered as a low-pass thermal filter: the periodic current that is injected in the resistive path excites thermal oscillations that are filtered due to the thermal inertia of the system. The driving efficiency depends obviously on the cutoff frequency of this filter.

Numerical simulations by the finite elements method ${ }^{40}$ were performed to evaluate the efficiency of our design. An example is shown in Figure 4 for a cantilever with a resonance frequency of $100 \mathrm{MHz}$ and $Q=10$ or 100 .

For a driving voltage amplitude of $0.1 \mathrm{~V}$, one gets a peak-to-peak amplitude at resonance of $17.3 \mathrm{pm}(172 \mathrm{pm})$ for $Q=10(Q=100)$. The corresponding temperature elevation is below $0.5 \mathrm{~K}$ everywhere in the cantilever. This amplitude is largely sufficient for nc-AFM application, especially considering that it scales linearly with the $Q$ factor, which generally will be of the order of 1000 or higher. A cantilever with a similar geometry, with a resonance frequency of 1.823 $\mathrm{MHz}$ and $Q=10$ was also simulated. The peak-to-peak amplitude was $88 \mathrm{pm}$ for a $0.1 \mathrm{~V}$ drive.

The thermal power $P$ injected in the cantilever is given by

$$
P \alpha\left[V_{e x c} \cos \left(\omega_{e x c} t\right)\right]^{2}=\frac{V_{e x c}^{2}}{2}[1+\cos (\omega t)],
$$

where $\omega=2 \omega_{\text {exc }}$. For small enough oscillations, the system behaves linearly and the trajectory of the cantilever is the superposition of a static deflexion $\Delta z$ and an oscillation at $\omega$ 


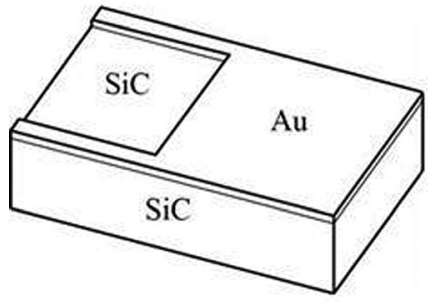

(a)

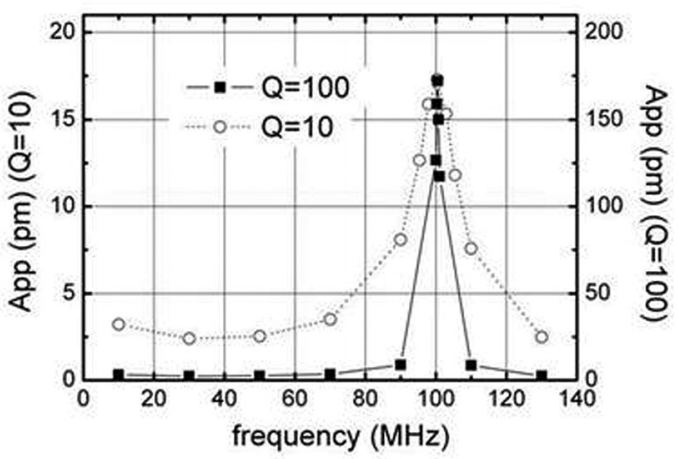

(b)

$$
z(t)=\Delta z+A \cos (\omega t) .
$$

When $\omega$ is well below the mechanical resonance $\omega_{0}$ and the thermal cutoff frequency $\omega_{\text {therm, }}$ one expects $\Delta z=A$, since both these quantities should be proportional to $V_{\mathrm{exc}}^{2}$, with the same proportionality coefficient. When $\omega=\omega_{0}$, the mechanical resonance of the cantilever, $z$ is enhanced by the quality factor $\mathrm{Q}$. But conversely, $\mathrm{z}$ is reduced by the thermal filter. If we consider that this filter is of first order, ${ }^{33}$ we get

$$
\frac{A}{\Delta z}=\frac{Q}{\sqrt{1+\frac{\omega_{0}^{2}}{\omega_{\text {therm }}^{2}}}},
$$

from which

$$
\frac{\omega_{\text {therm }}}{\omega_{0}}=\left[\left(\frac{Q \Delta z}{A}\right)^{2}-1\right]^{-\frac{1}{2}} .
$$

The values obtained by numerical simulations for the three examples presented in Figure 4 are gathered in Table IV. It is observed that the thermal cutoff frequency is approximately equal to the resonance frequency divided by two for the three cantilevers.

\section{Final design}

The cantilevers with different resonance frequency were designed in order to integrate the thermal excitation and the piezoresistive circuits. The support chip shown in Fig. 5(a) has the standard dimensions of commercial AFM cantilevers allowing the fixing on standard scanning probe microscopes heads. The four electrodes necessary for the electrical

TABLE IV. Results of the simulation of the electrothermal excitation. A: amplitude at resonance, $\Delta z$ : static deflexion, $\omega_{\text {therm }}$ thermal cutoff frequency.

\begin{tabular}{lccc}
\hline \hline & $\begin{array}{c}f_{0}=100 \mathrm{MHz}, \\
\mathrm{Q}=100\end{array}$ & $\begin{array}{c}f_{0}=100 \mathrm{MHz}, \\
\mathrm{Q}=10\end{array}$ & $\begin{array}{c}f_{0}=1.823 \mathrm{MHz}, \\
\mathrm{Q}=10\end{array}$ \\
\hline $\mathrm{A}(\mathrm{pm})$ & 86 & 8.6 & 88 \\
$\Delta z(\mathrm{pm})$ & 2.2 & 2.2 & 20 \\
$A / \Delta \mathrm{z}$ & 39 & 3.9 & 4.4 \\
$\omega_{\text {therm }} / \omega_{0}$ & 0.42 & 0.42 & 0.49 \\
\hline \hline
\end{tabular}

contacting of the two circuits are deposited on this support. The active part of the piezoresistive circuit is made of two branches of length $L_{m}$ varying from $1 \mu \mathrm{m}$ to $4 \mu \mathrm{m}$ and width $\mathrm{W}_{\mathrm{m}}$ varying from $100 \mathrm{~nm}$ to $400 \mathrm{~nm}$ depending on the overall size of the cantilever (Figure 5(b)). These branches were positioned at the base of the cantilever in the region where the strain is maximal. The dimensions of the whole piezoresisitive circuit were calculated to reach a total resistance of the circuit of $50 \mathrm{Q}$ with a thickness of gold of $30 \mathrm{~nm}$ and a conductivity $\sigma=\sigma_{\mathrm{Au}} / 3$. The width $W_{\text {exc }}$ of the thermal excitation circuit is twice that of the piezoresistive branches.

\section{FABRICATION OF THE CANTILEVERS}

\section{A. 3C-SiC films elaboration}

The 3C-SiC films for cantilever fabrication were grown via Chemical Vapor Deposition (CVD) on on-axis oriented (100) silicon wafers in a resistively heated horizontal hot wall CVD reactor. ${ }^{34}$ Standard gas system was used with silane $\left(\mathrm{SiH}_{4}\right)$ and propane $\left(\mathrm{C}_{3} \mathrm{H}_{8}\right)$ as precursor gases and purified hydrogen $\left(\mathrm{H}_{2}\right)$ as carrier gas. Previously described two-step process (substrate carbonization followed by CVD growth) was applied. ${ }^{35}$ Taking into account very low target thickness $(500 \mathrm{~nm})$ of $3 \mathrm{C}-\mathrm{SiC}$ film, the standard precursors flow rates were reduced and the growth rate was decreased to $\sim 1.5 \mu \mathrm{m} / \mathrm{h}$ in order to correctly control the film deposition.

Highly resistive $(\rho>10000 \Omega \mathrm{cm})$, p-type doped, Float Zone (FZ) Si wafers of $100 \mathrm{~mm}$ diameter served as substrates. The substrates were rotated during the growth in order to improve the thickness uniformity of the film: the thickness dispersion of the as-grown 3C-SiC layer, determined from Fourier transform infrared reflectance spectrometry measurements, was in the range of $2 \%-5 \%$.

Crystalline quality of 3C-SiC material was verified by $\mathrm{X}$-Ray Diffraction (XRD) measurements of the rocking curve of $3 \mathrm{C}-\mathrm{SiC}(002)$ diffraction peak, performed at different points along the diameter of the epiwafer. The Full Width at Half Maximum (FWHM) of the $\omega$ - scan was constant, $\sim 2200$ arc sec, except for the points at less than $5 \mathrm{~mm}$ from the epiwafer border confirming structural uniformity of the deposited material. The relatively high value of $\mathrm{SiC}$ (002) FWHM is nevertheless typical for the state of the art $3 \mathrm{C}-\mathrm{SiC}$ material in the studied thickness range. ${ }^{36}$ The 


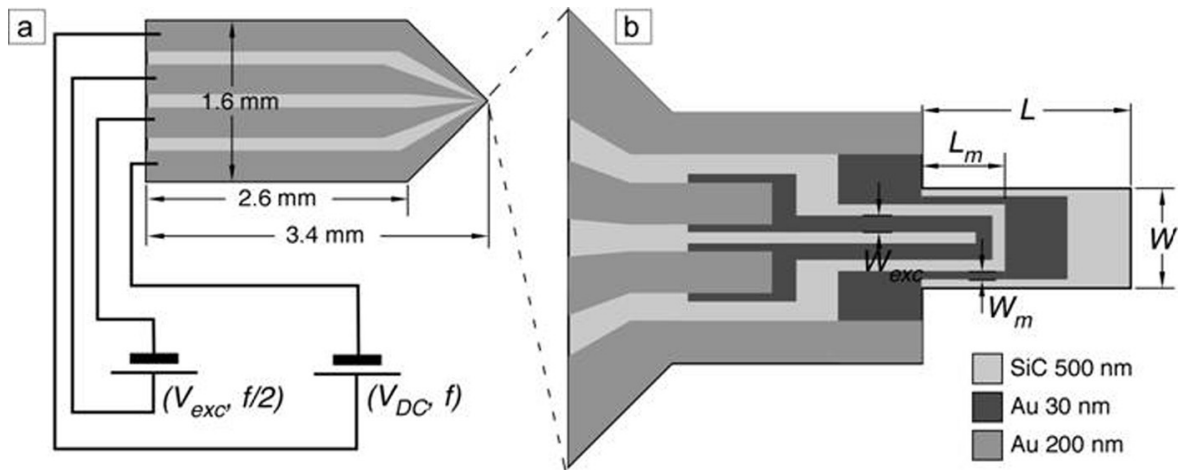

FIG. 5. (a) Size of the support chip and drawing of the electrical circuits for excitation and piezoresistive measurement of the $\mathrm{SiC}$ cantilever, (b) drawing of the $\mathrm{SiC}$ cantilever with the active circuits.

epiwafer bow, determined from the evolution of the $\mathrm{SiC}$ (002) maximum along the wafer diameter, was lower than $20 \mu \mathrm{m}$.

The as-grown 3C-SiC films presented a typical morphology for on-axis 3C-SiC(100) material with a characteristic pattern of anti-phase domains (Fig. 6(a)). The film roughness, determined using AFM, was in the range of $8-9 \mathrm{~nm}$. It was previously shown that high $3 \mathrm{C}-\mathrm{SiC}$ film roughness may result in worse quality of metallic contacts. In order to improve the surface quality, the epilayers were planarized using Chemical-Mechanical Polishing (CMP). ${ }^{37}$ An important planarization related issue was to preserve the low initial thickness dispersion of very thin $3 \mathrm{C}-\mathrm{SiC}$ layer. After the optimization of CMP process, the film roughness could be reduced down to $\sim 1 \mathrm{~nm}$ (Fig. 6(b)) after a homogeneous removal of $\sim 0.3 \mu \mathrm{m}$ of $3 \mathrm{C}-\mathrm{S} 1 \mathrm{C}$.

The growth conditions chosen for the heteroepitaxial film deposition have an important influence on the residual stress of grown material. These correlations were intensively studied and analytical model of stress relaxation was proposed. Detailed information can be found in Ref. 38 .

\section{B. Fabrication of the cantilevers}

The process to fabricate the high frequency cantilevers consists in three steps of e-beam lithography followed by a wet etching step to release the cantilever (Fig. 7(a)). The ebeam lithography machine is a "VISTEC EBPG 5000 plus" with a beam energy of $100 \mathrm{keV}$. The thin films $\mathrm{Cr} / \mathrm{Au}$ layers were evaporated using electron beam physical vapor deposition (EBPVD) in a PLASSYS MEB550 evaporator with a base pressure of $6 \times 10^{-8}$ mbars and a bias voltage of

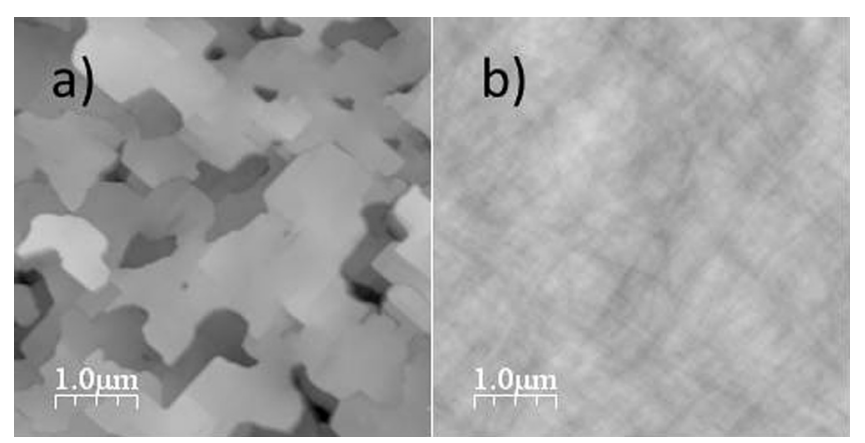

FIG. $6.5 \times 5 \mu \mathrm{m}^{2}$ AFM images of $500 \mathrm{~nm}$ thick $3 \mathrm{C}$-SiC films: (a) as-grown film (z range $80 \mathrm{~nm}$, RMS roughness $8 \mathrm{~nm}$ ); (b) $800 \mathrm{~nm}$ thick $3 \mathrm{C}-\mathrm{SiC}$ film CMP planarized down to $500 \mathrm{~nm}$ (z range $20 \mathrm{~nm}$, RMS roughness $0.8 \mathrm{~nm}$ ).
$10 \mathrm{keV}$. The lift-off steps were carried in trichloroethylene heated at $50^{\circ} \mathrm{C}$. The first step of the process is the fabrication of the thin circuits of $\mathrm{Cr} / \mathrm{Au}(0.7 \mathrm{~nm} / 30 \mathrm{~nm})$ with PMMA resist and lift-off. The second step is the fabrication of the thick $\mathrm{Cr} / \mathrm{Au}(5 \mathrm{~nm} / 200 \mathrm{~nm})$ electrodes with PMMA resist and lift-off. We performed electrical I(V) measurements of the gold circuits using an AGILENT 41501B Pulse Generator with 4SMU driven by ICS/4155C software. I(V) characteristics were obtained by varying the input voltage from $-0.5 \mathrm{~V}$ to $+0.5 \mathrm{~V}$. After optimization of the thicknesses of the $\mathrm{Cr} / \mathrm{Au}$ active circuits, the resistances of the piezoresistive circuits were between 45 and $52 \Omega$.

The third step of the process is the etching of the $\mathrm{SiC}$ $500 \mathrm{~nm}$ thick layer. The ma-N2410 photoresist was lithographed by e-beam and the etching of $\mathrm{SiC}$ was done by reactive ion plasma etching (RIE) with $\mathrm{S} \mathrm{F}_{6}$ gas. The final step is the release of the cantilever by wet anisotropic etching of $\mathrm{Si}$ using a tetramethyl-ammonium-hydroxyde (TMAH) solution at $80^{\circ} \mathrm{C}$. Simulation of the cantilever oscillations has shown that the anchoring of the cantilever is an important parameter: the presence of underetching below the base that supports the cantilever can degrade strongly the quality factor of the cantilever. As the etching rate of Si by TMAH is lower on the (111) plane than on the (100) plane, the cantilevers were aligned along the $\mathrm{Si}\{100\}$ direction to better control this underetching. Figure 7(b) shows a SEM image of a 14 um long cantilever of $5 \mathrm{MHz}$ resonance frequency.

\section{MEASUREMENTS}

The measurements were performed at room temperature and under vacuum $\left(P \sim 10^{-7}\right.$ Torr $)$ with a voltage preamplifier from FEMTO, ${ }^{41}$ with a bandwidth of $200 \mathrm{MHz}$ and a voltage input noise of approximately $1.2 \mathrm{nV} . \mathrm{Hz}^{-\frac{1}{2}}$ and a lockin amplifier from Stanford Research Systems, ${ }^{42}$ with a bandwidth of $200 \mathrm{MHz}$. The modulus and phase of the frequency response of two typical cantilevers are displayed in Figure 8. The black curves are the Lorentzian fit of the experimental curves with the parameters indicated in the figure. The quality of the adjustment is very good showing that the cantilevers behave as harmonic oscillators near their resonance frequency.

Note that the data leading to the curves of Figure 8 have been corrected by subtraction of a parasitic signal. This signal has its origin in the finite value of $\alpha=\frac{\Delta R}{R \Delta T}$, the temperature coefficient of resistivity of metals. Due to the thermal 


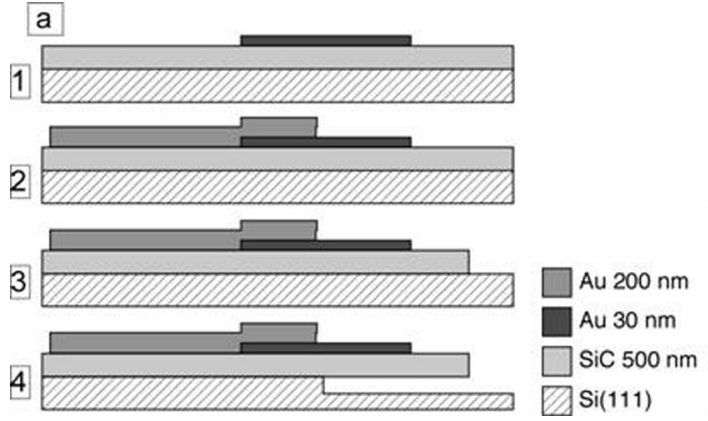

coupling via the $\mathrm{SiC}$ body of the cantilever, the temperature of the piezoresistor is modulated by $\Delta T$ at the oscillation frequency. As a consequence, its resistance is modulated according to $\frac{\Delta R}{R}=\alpha \Delta T$. For $\mathrm{Au}$ near room temperature, $\alpha \approx$ $4 \times 10^{-4} \mathrm{~K}^{-1}$. The simulations presented in Sec. III C, suggest that $\Delta T$ should be below $0.5 \mathrm{~K}$ at the position of the piezoresistance, giving $\frac{\Delta R}{R_{\mathrm{ther}}} \approx 2 \times 10^{-4}$. The relative modulation of the piezoresistance at resonance for the $Q=100$ cantilever of Figure $4(\mathrm{~b})$ is $\frac{\Delta R}{R \text { strain }}=4 \times 10^{-5}$, showing that this parasitic signal can be large compared to the signal of interest. But as $\frac{\Delta R}{R}$ strain $/ \frac{\Delta R}{R}$ therm is proportional to $Q$, this offset signal should be much smaller for higher values of $Q$. In any case, as this signal is deterministic, it is possible to subtract it from the data by simple electronic means. Indeed, we developed an alldigital phase-locked loop (PLL) which incorporates such a compensation scheme, in addition to frequency doubling at the desired resonance frequencies of the fabricated cantilevers. $^{43}$

The results of the measurements performed on a family of cantilevers are summarized in Table III. The measured resonance frequencies are largely below the calculated ones. This discrepancy has 2 origins: (1) The presence of the Au circuits, which is not taken into account in the calculation, lowers $f_{0}$ because of the lower Young's modulus and higher density of $\mathrm{Au}$ relative to SiC. (2) A Young's modulus $E=440 \mathrm{GPa}$ was introduced in the calculation, but there is evidence for a much smaller value in $\mathrm{SiC}$ thin films, due to a defect density higher than in the bulk, as measured by some of us who got a value $E \approx 350 \mathrm{GPa} .{ }^{17}$ Another factor of uncertainty in the resonance frequency is due to the difficulty in controlling underetching effects mentioned in Sec. IV. This behaviour is confirmed by the fact that the values of $f_{0}$ and $Q$ are much less dispersed for cantilevers that have been etched simultaneously.

It is seen that $Q$ decreases rapidly with the resonance frequency $f_{0}$, being approximately proportional to $1 / f_{0}$. This behaviour is frequently observed in MEMS or NEMS cantilevers and is generally considered as characterizing a damping mechanism due to attachment losses: ${ }^{44-46}$ As mentioned previously, the strain is concentrated near the clamping point of the cantilever and this time-varying strain radiates elastic energy into the support. It is not possible to give a precise interpretation of these measurements as the clamping geometry of our cantilevers is somewhat ill-defined due to the underetching problems mentioned previously.

It was checked that the amplitude of the oscillation is linear in $V_{D C}$, as indicated by expression (3) and quadratic in $V_{\text {exc }}$, as discussed in Sec. III C.

The influence of the pressure $P$ on the $Q$ factor of a few cantilevers was measured. As previously reported, ${ }^{15,47} Q$ is constant at low pressure; it decreases as $1 / P$ in an intermediate molecular flow regime before entering the viscous
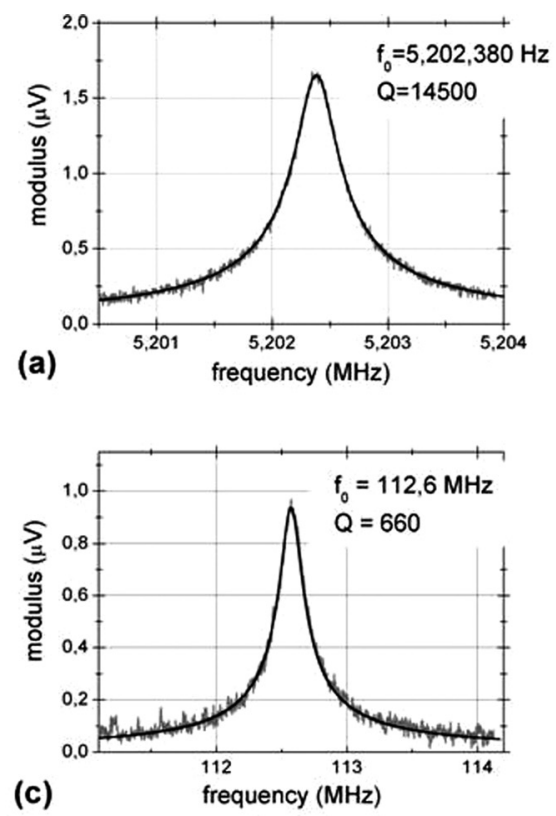
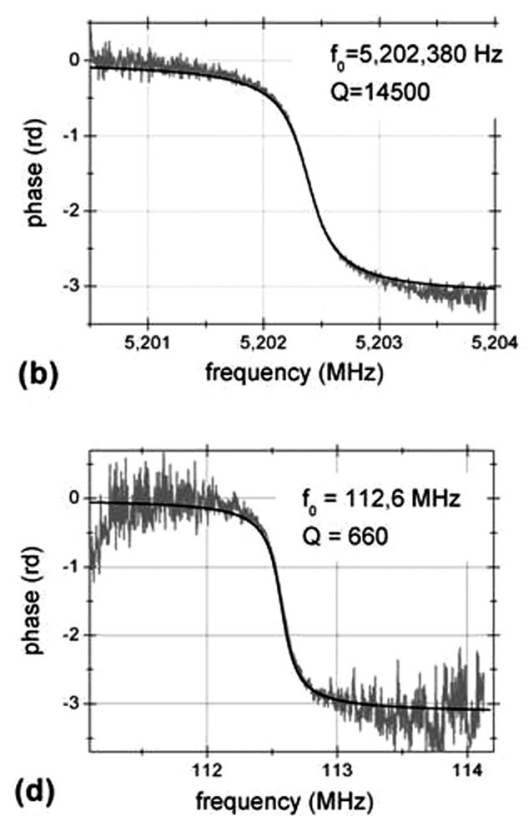

FIG. 8. (a) and (c) modulus and (b) and (d) phase of the response for two cantilevers (gray curves). Conditions: under vacuum, $V_{D C}=100 \mathrm{mV}$, $V A_{C}=100 \mathrm{mV}_{p p}$. The frequency axis gives $f=2 f_{\text {exc }}$. The black lines are the Lorentzian fit of the experimental curves with the parameters indicated in the figure. 
damping regime. This transition happens at higher pressure for higher frequency cantilevers, because $Q$ is less lowered by viscous damping in air for smaller cantilevers. The best $Q$ factors we got in air are of the order of 500 .

\section{CONCLUSION}

The main result of this work is the demonstration that driving a $\mathrm{SiC}$ cantilever at frequency as high as $100 \mathrm{MHz}$ by thermoelastic excitation is compatible with the piezoresistive method. A major advantage of this method is related to the $50 \Omega$ impedance of the piezoresistance. It makes the readout electronics much simpler. It also makes the device less sensitive to noise and allow, in particular, to implement the preamplifier at a large distance from the cantilever without a significant degradation of the signal, a major advantage for in-vacuum applications. The two drawbacks of the thermoelastic excitation are

(1) It heats the cantilever, but this is by an amount that is comparable to the unavoidable heating induced by the piezoresistive detection. Furthermore, the simulations show that the temperature rise of the cantilever stays below $1 \mathrm{~K}$ in our experimental conditions.

(2) It generates a parasitic signal, due to the finite temperature coefficient of resistivity of the piezoresistor. We are confident that this signal can be minimized by optimizing the design of the cantilevers. In addition, it can be compensated by simple electronic means. ${ }^{43}$

It is possible to evaluate the measurement noise of our cantilevers from expression (5), but this requires to measure the piezoresisitive gauge factor $\gamma$. Expression (3) can be used for that purpose. The amplitude of vibration could be very roughly estimated from SEM images of a cantilever excited at its resonance frequency of $1.146 \mathrm{MHz}$. A value $\gamma_{\text {exp }} \sim 5$ could be deduced from this measurement. It is significantly higher but of the same order of magnitude than the expected value $\gamma_{\exp } \sim 3$. Injecting it in expression (5), with $V_{D C}=0.1 \mathrm{~V}$ and the parameters of Tables I and II give $\left[S_{z}(\omega)\right]^{\frac{1}{2}}=3.3 \mathrm{pm} \cdot \mathrm{Hz}^{-\frac{1}{2}}$ for $f_{0}=1 \mathrm{MHz}$ and $\left[S_{z}(\omega)\right]^{\frac{1}{2}}=33 \mathrm{fm} \cdot \mathrm{Hz}^{-\frac{1}{2}}$ for $f_{0}=100 \mathrm{MHz}$. This last value is comparable to the best ones reported in the literature, ${ }^{2,18,21}$ demonstrating that this type of cantilever could be competitive at least for high resonance frequencies.

In Ref. 15, the $Q$ values reported for $\mathrm{SiC}$ cantilevers with resonance frequency in the $1 \mathrm{MHz}-127 \mathrm{MHz}$ range are nearly constant, around $Q \approx 1000$. This contrasts with our values measurements, where $Q$ decreases by more than an order of magnitude from much higher $(Q \sim 11000-15000)$ at the lowest frequency. One possible explanation invokes the defect density in the SiC epilayers. It is known that the defect density in this type of films is rather high and decreases with the distance from the $\mathrm{Si}-\mathrm{SiC}$ interface. ${ }^{48}$ It is then likely that the defect density in our films $(t=500 \mathrm{~nm})$ is lower than in the films of Ref. $15(t=100 \mathrm{~nm})$ contributing to a better $Q$. This would indicate that the dominant dissipation mechanism in Ref. 15 is related to bulk or surface defects $^{44,45}$ rather than clamping losses, as in our case. In addition, the SiC epilayers used in this work were CMP planarized, as described in Sec. IV. This treatment results in a better surface quality, which could also explain a better $Q$.

The decrease of $Q$ at higher frequency is a general problem for high frequency NEMS, which has not received a simple general solution. We have then to rely on detailed studies of different geometry of attachment of the cantilever on its support to maximize the impedance mismatch between the two parts as suggested in Ref. 49.

To use these cantilevers on an AFM head, it will be necessary to add a tip near their extremity. The growth of Si on $\mathrm{SiC}$ epilayer has been optimized in the perspective of making Si tips and a process based on dry etching has been developed by some of us as described in Ref. 50.

\section{ACKNOWLEDGMENTS}

This work has been supported by the Agence Nationale de la Recherche (ANR) in the frame of its program in Nanosciences and Nanotechnologies (Nanosens Project No. ANR-08-NANO-017). The help of C. Deshayes, E. Dujardin, A. Miranda, and C. Soldano during this project is gratefully acknowledged.

${ }^{1}$ G. Meyer and N. M. Amer, Appl. Phys. Lett. 53, 1045 (1988).

${ }^{2}$ T. Fukuma et al., Rev. Sci. Instrum. 76, 053704 (2005).

${ }^{3}$ D. Rugar, H. J. Mamin, and P. Guethner, Appl. Phys. Lett. 55, 2588 (1989).

${ }^{4}$ M. Tortonese, R. Barrett, and C. Quate, Appl. Phys. Lett. 62, 834 (1993).

${ }^{5}$ O. Hansen and A. Boisen, Nanotechnology 10, 51 (1999).

${ }^{6}$ J. A. Harley and T. W. Kenny, Appl. Phys. Lett. 75, 289 (1999).

${ }^{7}$ J. L. Arlett, J. R. Maloney, B. Gudlewski, M. Muluneh, and M. L. Roukes, Nano Lett. 6, 1000 (2006).

${ }^{8}$ S. Watanabe and T. Fujii, Rev. Sci. Instrum. 67, 3898 (1996).

${ }^{9}$ S. C. Minne, S. R. Manalis, A. Atalar, and C. F. Quate, Appl. Phys. Lett. 68, 1427 (1996).

${ }^{10}$ K. Bartzke, T. Antrack, K.-H. Schmidt, E. Dammann, and C. H. Schatterny, Int. J. Optoelectron. 8, 669 (1993).

${ }^{11}$ F. J. Giessibl, Appl. Phys. Lett. 73, 3956 (1998).

${ }^{12}$ T. Akiyama, U. Staufer, N. F. de Rooij, P. Frederix, and A. Engel, Rev. Sci. Instrum. 74, 112 (2003).

${ }^{13}$ X. M. H. Huang, C. A. Zorman, M. Mehregany, and A. L. L. Roukes, Nature 421, 496 (2003).

${ }^{14}$ T. Ando, Nanotechnology 23, 062001 (2012).

${ }^{15}$ M. Li, H. X. Tand, and M. L. Roukes, Nat. Nanotechnol. 2, 114 (2007).

${ }^{16}$ Y. T. Yang, K. L. Ekinci, X. M. H. Huang, L. M. Schiavone, M. L. Roukes, C. A. Zorman, and M. Mehregany, Appl. Phys. Lett. 78, 162 (2001).

${ }^{17}$ J.-F. Michaud, S. Jiao, A. E. Bazin, M. Portail, T. Chassagne, M. Zielinski, and D. Alquier, Silicon Carbide 2010 Materials, Processing and Devices, Mater. Res. Soc. Symp. Proc. 2010, Vol. 1246, p. B09-04.

${ }^{18}$ F. J. Giessibl, Rev. Mod. Phys. 75, 949 (2003).

${ }^{19}$ T. R. Albrecht, P. Grütter, D. Home, and D. Rugar, J. Appl. Phys. 69, 668 (1991).

${ }^{20}$ J. Polesel-Maris, M. A. Venegas de la Cerda, D. Martrou, and S. Gauthier, Phys. Rev. B 79, 235401 (2009).

${ }^{21}$ J. Lübbe, M. Temmen, P. Rahe, A. Kühnle, and M. Reichling, Beilstein J. Nanotechnol. 4, 32 (2013).

${ }^{22}$ F. Pielmeier, D. Meuer, D. Schmid, C. Strunk, and F. J. Giessibl, Beilstein J. Nanotechnol. 5, 407 (2014).

${ }^{23}$ F. J. Giessibl, H. Bielefeld, S. Hembacher, and J. Mannhart, Appl. Surf. Sci. 140, 352 (1999).

${ }^{24}$ D. Sarid, Scanning Force Microscopy (Oxford University Press, 1991).

${ }^{25}$ Y. Song and B. Bhushan, Microsyst. Technol. 12, 219 (2006).

${ }^{26}$ Product Guide Nanosensors, 2001.

${ }^{27}$ S. Enderling et al., J. Micromech. Microeng. 17, 213 (2007).

${ }^{28}$ S. U. Jen et al., Thin Solid Films 434, 316 (2003).

${ }^{29}$ G. C. Ratcliff, D. A. Erie, and R. Superfine, Appl. Phys. Lett. 72, 1911 (1998). 
${ }^{30}$ A. Labuda, K. Kobayashi, D. Kiracofe, K. Suzuki, P. H. Grütter, and H. Yamada, AIP Adv. 1, 022136 (2011).

${ }^{31}$ R. Pedrak, T. Ivanov, K. Ivanova, T. Gotszalk, N. Abedinov, I. W. Rangelow, K. Edinger, E. Tomerov, T. Schenkel, and P. Hudek, J. Vac. Sci. Technol. B 21, 3102 (2003).

${ }^{32}$ L. Jiang, R. Cheung, J. Hedley, M. Hassan, A. J. Harris, J. S. Burdess, M. Mehregany, and C. A. Zorman, Sens. Actuators, A 128, 376 (2006).

${ }^{33}$ T. S. J. Lammerink, M. Elwenspoek, and J. H. Fluitman, Sens. Actuators, A 27, 685 (1991)

${ }^{34}$ A. Leycuras, Mater. Sci. Forum 338-342, 241 (2000).

${ }^{35}$ M. Portail, M. Zielinski, T. Chassagne, S. Roy, and M. Nemoz, J. Appl. Phys. 105, 083505 (2009).

${ }^{36}$ M. Zielinski, M. Portail, S. Roy, T. Chassagne, C. Moisson, S. Kret, and Y. Cordier, Mater. Sci. Eng. B 165, 9 (2009).

${ }^{37}$ M. Zielinski, C. Moisson, S. Monnoye, H. Mank, T. Chassagne, S. Roy, A. E. Bazin, J. F. Michaud, and M. Portail, Mater. Sci. Forum 645-648, 753 (2010).

${ }^{38}$ M. Zielinski, J. F. Michaud, S. Jiao, T. Chassagne, A. E. Bazin, A. Michon, M. Portail, and D. Alquier, J. Appl. Phys. 111, 053507 (2012).
${ }^{39}$ A. C. Hilliera and A. J. Bard, Rev. Sci. Instrum. 68, 2082 (1997).

${ }^{40}$ Comsol Multiphysics, Comsol Inc., USA.

${ }^{41}$ HVA-200M-40-B from FEMTO Messtechink GmbH, Berlin, Germany.

${ }^{42}$ SR844 from Stanford Research Systems, Sunnyvale, CA, USA.

${ }^{43}$ J. Bouloc, L. Nony, C. Loppacher, W. Rahajandraibe, F. Bocquet, and L. Zaid, in IEEE Sensors, Taipei, 28-31 October (2012), pp. 1-4.

${ }^{44}$ X. M. H. Huang, C. A. Zorman, M. Mehregany, and M. L. Roukes, in Proceeding of the 12th International Conference on Solid-State Sensors, Actuators and Microsystems, Boston, TRANSDUCERS '03 (2003).

${ }^{45}$ X. M. H. Huang, X. L. Feng, C. A. Zorman, M. Mehregany, and M. L. Roukes, New J. Phys. 7, 247 (2005).

${ }^{46} \mathrm{M}$. Imboden and P. Mohanty, Phys. Rep. 534, 89-146 (2014).

${ }^{47}$ W. E. Newell, Science 161, 1320 (1968).

${ }^{48}$ X. Song, J. F. Michaud, F. Cayrel, M. Zielinski, M. Portail, T. Chassagne, E. Collard, and D. Alquier, Appl. Phys. Lett. 96, 142104 (2010).

${ }^{49}$ J. Rieger, A. Isacsson, M. J. Seitner, J. P. Kotthaus, and E. M. Weig, Nat. Commun. 5, 3345 (2014).

${ }^{50}$ S. Jiao, J. F. Michaud, M. Portail, A. Madouri, T. Chassagne, M. Zielinski, and D. Alquier, Mater. Lett. 77, 54 (2012). 